\title{
Os Benefícios da Ressecção Anterior Baixa em Monobloco para o Câncer de Ovário Avançado: Dez Anos de Experiência em um Único Centro Terciário
}

\author{
The Benefits of Low Anterior in Bloc Resection for Advanced Ovarian Cancer: \\ Ten-Year Experience in a Single Tertiary Center
}

\author{
SERGIO RENATO PAIS COSTA ${ }^{1} ;$ RENATO ARIONILUPINACCI $^{2}$ \\ ${ }^{1}$ Especialista em Cancerologia Cirúrgica e Cirurgia do Aparelho Digestivo, Assistente do Serviço de Cirurgia Geral e \\ Oncocirurgia do Hospital do Servidor Público Estadual de São Paulo - "Francisco Morato de Oliveira", Mestre em \\ Cirurgia pela Universidade Federal de São Paulo - UNIFESP-EPM, São Paulo, Brasil; ${ }^{2}$ Especialista em \\ Coloproctologia, Diretor do Serviço de Cirurgia Geral e Oncocirurgia do Hospital do Servidor Público Estadual de \\ São Paulo - "Francisco Morato de Oliveira", Doutor em Cirurgia pela Universidade Federal de São Paulo - UNIFESP- \\ EPM, São Paulo, Brasil.
}

COSTA SRP; LUPINACCI RA. Os Benefícios da Ressecção Anterior Baixa em Monobloco para o Câncer de Ovário Avançado: Dez Anos de Experiência em um Único Centro Terciário. Rev bras Coloproct, 2008;28(2): 160-169.

RESUMO: Introdução - A pedra angular do tratamento do câncer epitelial de ovário tem sido a associação da cirurgia citorredutora aliada à quimioterapia com platina. Quando essa neoplasia invade órgãos pélvicos adjacentes somente uma ressecção pélvica em monobloco pode levar a uma citorredução ótima. Objetivo - O objetivo do presente estudo foi acessar a segurança, a eficácia e o impacto na sobrevida da ressecção anterior baixa em monobloco para o câncer epitelial do ovário avançado. Métodos - Os dados completos de 19 pacientes que foram submetidos a esse tipo de operação para tratamento de câncer de ovário avançado entre Janeiro de 1996 a Junho de 2006 foram analisados. A mediana de idade foi de 58 anos (24-77). Em todas as cirurgias a doença residual foi menos do que $1 \mathrm{~cm}$. Resultados - A mortalidade pós-operatória foi de $5 \%$ (n=1) enquanto a morbidade foi de $26,3 \%$ $(n=5)$. A sobrevida global em três anos foi de 76,47 \% enquanto a mediana foi de 42 meses. Conclusões - A ressecção anterior baixa em monobloco não deve ser um obstáculo quando uma citorredução ótima é necessária. Em que pese sua alta morbidade, sua mortalidade é aceitável. $O$ controle da doença em longo prazo pode ser atingido com esta prática.

Descritores: Exenteração Pélvica, Câncer de Ovário, Morbidade, Mortalidade, Resultados do Tratamento.

\section{INTRODUÇÃO}

Aproximadamente 14,300 mulheres faleceram devido ao câncer de ovário em 2003, nos Estados Unidos. Neste país, esta neoplasia representa a principal causa de morte por câncer ginecológico. Em linhas gerais, o câncer de ovário corresponde respectivamente a 4\% de todos os diagnósticos de câncer e cerca de $5 \%$ de todos os óbitos por câncer. Em estádios precoces é freqüentemente curável. Infelizmente, 60-70 \% das pacientes apresentam-se em um estádio avançado no momento de seu diagnóstico. A despeito do fato de que a maioria das pacientes responde $(80 \%)$ inicialmente a quimioterapia, a maior parte sucumbe em virtude da enfermidade. Os índices de sobrevida global em cinco anos nos estádios avançados têm se situado em torno de $10 \%$. Quando um tumor de ovário avançado é descoberto durante a avaliação intra-operatória, a conduta padronizada é um adequado estadiamento cirúrgico associado a uma citorredução agressiva. A

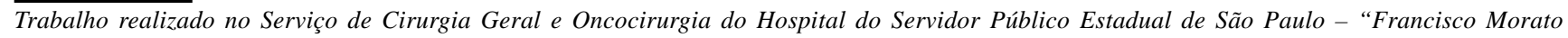
de Oliveira". 
citorredução refere-se à remoção de toda doença possível. Enquanto que para a maioria dos tumores sólidos, a ressecção cirúrgica agressiva somente se justifica quando todo tumor pode ser removido oferecendo uma cirurgia potencialmente curativa. Ao contrário, para o câncer de ovário, a citorredução cirúrgica quando a doença residual é menor que $1 \mathrm{~cm}$ (citorredução ótima) pode oferecer maiores índices de resposta à quimioterapia, intervalos mais longos sem progressão da doença e aumento na mediana de sobrevida. ${ }^{1-4}$

Tanto a definição de citorredução ótima quanto a porcentagem de pacientes em estádios avançados em que possa ser realizada essa abordagem, tem variado amplamente na literatura. A definição de citorredução ótima tem variado desde doença não visível até 0,5 ou $1 \mathrm{~cm}$ de doença residual.

A porcentagem de pacientes em estádios clínicos III-IV que podem ser submetidos a uma citorredução ótima tem variado de $25 \%$ a $75 \%$ dos casos. Desta maneira, a citorredução ótima não pode ser atingida em uma proporção significativa das doentes, principalmente nos tumores avançados. Por sua vez, quando esses tumores invadem estruturas pélvicas adjacentes (peritônio pélvico, fundo de saco, bexiga, reto e cólon sigmóide) para se atingir uma citorredução ótima, a solução tática é a realização de uma ressecção anterior baixa associada a uma ressecção em monobloco dos demais órgãos pélvicos envolvidos (exenteração pélvica). ${ }^{5-9}$

Essa conduta cirúrgica, no entanto apresenta alta morbidade aliada a substancial mortalidade. Quando somente o reto ou cólon sigmóide em sua porção inferior é invadido, a ressecção anterior baixa com histerectomia total em monobloco também designada como exenteração pélvica posterior pode ser o suficiente. Contudo, quando ocorre à invasão conjuntamente do trígono vesical, o procedimento padronizado é a ressecção dos órgãos do trato urinário inferior (bexiga e terço distal dos ureteres) e do trato genital interno associado à ressecção anterior baixa em monobloco (exenteração pélvica total supra-elevadora) ${ }^{10} \mathrm{Como}$ alternativa estratégica, com o objetivo de se evitar cirurgias multiviscerais, a utilização da quimioterapia neoadjuvante tem sido recomendada em associação a citorredução de intervalo. ${ }^{11-12}$

Embora existam poucas séries descritas na literatura sobre o uso dessa operação no tratamento do câncer de ovário, especificamente no Brasil não encontramos nenhuma.
Assim sendo, o objetivo do presente estudo foi avaliar os resultados precoces e tardios (morbidade, mortalidade e sobrevida) das pacientes com câncer epitelial de ovário avançado submetidos a esse tipo de operação como parte de uma cirurgia citorredutora ótima, em uma experiência de dez anos em nosso serviço.

\section{MÉTODOS}

Entre Janeiro de 1996 e Junho de 2006, 37 doentes com câncer epitelial de ovário avançado (Estádios III-C e IV, FIGO) com envolvimento clínico e/ou radiológico de estruturas pélvicas (reto, cólon sigmóide, ureteres, bexiga) foram avaliados pelo Serviço Cirurgia Geral e Oncocirurgia do Hospital do Servidor Público Estadual de São Paulo, "Francisco Morato de Oliveira". Dentre essas doentes, um total de 19 foram submetidas à ressecção anterior baixa em monobloco (exenteração pélvica) e fizeram parte do presente estudo. Dezessete pacientes foram submetidas à ressecção anterior baixa do reto com histerectomia total em monobloco (exenteração pélvica posterior) e duas à ressecção anterior baixa do reto com histerectomia total, ureterectomia parcial bilateral e cistectomia total em monobloco (exenteração pélvica total supraelevadora). Os critérios de exclusão foram: câncer de ovário não-epitelial, tumores bordeline, citorredução secundária, doença recorrente, exenteração paliativa, citorredução não-ótima, cirurgias realizadas em outro departamento ou instituição.

Foram colhidos de maneira retrospectiva os seguintes dados de prontuário: idade, origem, doença associada, classificação do risco cirúrgico - ASA (American Society of Anesthesiology), estádio do tumor, subtipo histológico e grau, sintomas pré-operatórios, níveis séricos de CA 125, nível sérico pré-operatório de albumina, achados radiológicos pré-operatórios (tomografia de abdome e pelve), tipo de cirurgia, tipo de droga usada na quimioterapia, número de ciclos, resposta a quimioterapia, doença residual após citorredução, hemorragia estimada intra-operatória, tempo cirúrgico, morbidade, tempo de internação hospitalar, mortalidade pós-operatória, número de linfonodos dissecados, modo e tempo de recorrência, tempo de intervalo livre de doença, sobrevida global e sobrevida específica livre de doença.

Todas as pacientes foram estadiadas de acordo com as normas da International Federation of 
Gynecology and Obstetrics System. ${ }^{13}$ A definição de citorredução ótima adotada em nosso serviço tem sido a descrita pelo Gynecologic Oncology Group que considera como lesão residual menos do que $1 \mathrm{~cm}$ de doença. ${ }^{12}$

Primariamente, cinco doentes foram excluídas devido à má condição clínica pré-operatória sendo tratadas paliativamente apenas com suporte clínico. Consequientemente, das 32 restantes, dezessete já apresentavam critérios de irressecabilidade radiológica préoperatória, e foram encaminhadas para tratamento quimioterápico primário. Sendo assim, quinze foram exploradas cirurgicamente com intuito de avaliar sua ressecabilidade intra-operatória. Dessas quinze, em sete foi possível a realização de uma citorredução ótima primária (doença residual menor que $1 \mathrm{~cm}$ ). Nos demais $(n=8)$, a citorredução poderia ser ótima, contudo levaria a importante morbidade (reconstruções complexas tanto gastrointestinais quanto urinárias, múltiplas anastomoses gastrointestinais e ressecções mutiladoras). Assim sendo, essas doentes $(n=8)$ também seguidas das demais já consideradas irressecáveis de princípio (17) foram encaminhadas para quimioterapia neoadjuvante.

A conduta padronizada em nosso serviço em relação à quimioterapia neo-adjuvante tem sido: 1) se resposta parcial, a paciente é encaminhada a citorredução de intervalo; 2) se há ausência de resposta ou progressão de doença, a paciente é encaminhada a quimioterapia de segunda linha. A resposta é avaliada por exame clínico, nível sérico de CA 125 e tomografia computadorizada (TC). O padrão de resposta segue as determinações da WHO. ${ }^{(14)}$

Os regimes quimioterápicos utilizados foram todos baseados em platina associada nos casos mais antigos à ciclofosfamida $(\mathrm{n}=5)$ ou mais recentemente ao taxol $(n=20)$. A resposta à quimioterapia neoadjuvante foi avaliada depois do terceiro ciclo. Dezenove doentes apresentaram resposta parcial (índice de resposta de $76 \%$ ) e conseqüentemente foram submetidos à exploração cirúrgica. Doze seguiram citorredução ótima, todas associadas a exenteração (exenteração pélvica posterior $=10$ e exenteração pélvica total $=2$ ). Dois de nove que eram considerados irressecáveis antes da quimioterapia, se tornaram ressecáveis após o tratamento $(25 \%)$. A quimioterapia neoadjuvante rendeu uma citorredução ótima em doze doentes $(48 \%)$. Mesmo assim, sete pacientes permanecerão irressecáveis à avaliação intra-operatória.
Conseqüentemente, um total de 19 doentes apresentou tumor ressecável, sendo sete na cirurgia primária e doze na cirurgia de intervalo.

Ao término do tratamento todas as pacientes foram seguidas a cada três meses (durante dois anos), e a cada seis meses (entre dois e cinco anos). Em cada retorno foi realizado exame físico completo, CA 125 sérico, tomografias de abdome-pelve e raio-X de tórax. A recidiva foi somente considerada por exames de imagem confirmados com avaliação histológica.

\section{DESCRIÇÃO DA TÉCNICA CIRÚRGICA}

A exenteração pélvica total quando realizada seguiu os princípios técnicos já adotados em nosso Serviço e que foram previamente descritos. ${ }^{(10)} \mathrm{Em}$ contrapartida, os princípios técnicos da exenteração pélvica posterior (Figuras 1 e 2) seguiram aqueles descritos por Eisenkop et al. ${ }^{5}$ Previamente à ressecção pélvica radical, todos os doentes seguiram um procedi-

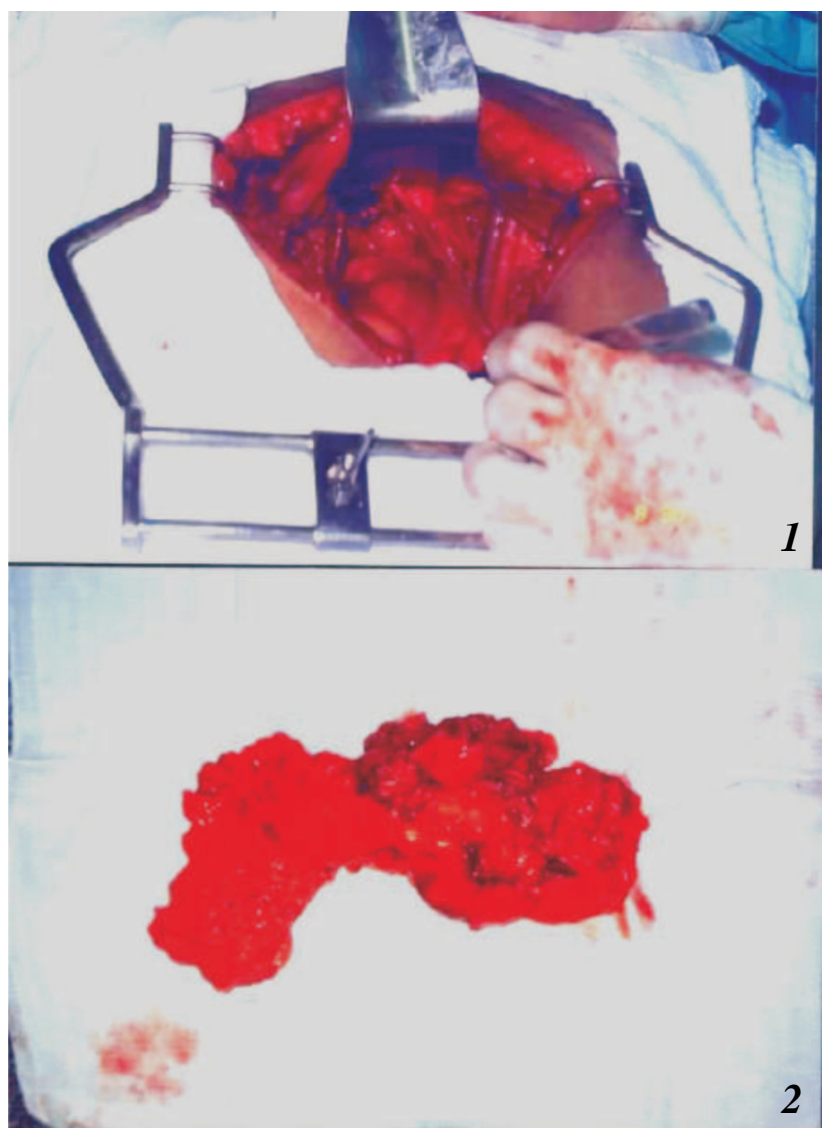

Figuras 1 e 2-Aspectos da pelve (foto acima) e da peça cirúrgica (foto abaixo) - ressecção em monobloco do reto-sigmóide com útero e anexos (Exenteração Pélvica Posterior Modificada). 
mento padronizado de estadiamento anatomo-cirúrgico conforme descrito pela FIGO como: coleta de lavado peritoneal abdominal ou pélvico (ou do líquido ascítico), omentectomia, linfadenectomia pélvica (ilíacoobturatória) e retroperitoneal (para-aórtica). As linfadenectomias tanto ilíaco-obturatória quanto paraaórtica seguiram os princípios técnicos descritos e adotados pelo Departamento de Oncoginecologia do Memorial Sloan Kettering Cancer (figuras 3 e 4). A apendicectomia somente foi realizada nos casos de adenocarcinoma mucinoso ou nos casos de comprometimento indubitável do apêndice cecal pela neoplasia. Todas as anastomoses colorretais foram mecânicas com o uso da técnica de duplo grampeamento. Em duas pacientes com idade avançada que apresentavam condição clínica de risco (ASA III), e em mais outras duas submetidas à exenteração pélvica total, a reconstrução utilizada foi uma colostomia terminal (tipo Hartman). Nessas duas últimas doentes a reconstrução urinária utilizada foi uma ureteroileostomia cutânea a Bricker.

\section{RESULTADOS}

Os achados clínicos, histológicos e laboratoriais estão demonstrados na Tabela 1. A mortalidade global da presente série foi de $5 \%(\mathrm{n}=1)$. O único óbito dessa série foi em uma paciente submetida à exenteração pélvica total que apresentou um grande abscesso pélvico. Essa doente foi reoperada, e faleceu por complicações infecciosas no trigésimo quinto dia de pósoperatório. Por outro lado, a morbidade da presente série foi de 26,3\% ( $n=5)$. Quinze doentes foram submetidas à pelo menos uma outra ressecção cirúrgica além da exenteração pélvica. (Cirurgias e Resultados estão demonstrados na Tabela 2).

O exame histológico revelou invasão do reto em todas doentes e invasão do trígono vesical em duas (até a mucosa). A serosa do reto foi invadida em cinco, enquanto a muscular foi invadida em dez. Quatro doentes apresentaram invasão até a mucosa. O número de linfonodos dissecados variou de 17 a 31 com mediana de 22. Nove doentes apresentaram linfonodos comprometidos (47\%). Quatro doentes apresentaram comprometimento da cadeia para-aórtica e duas da cadeia ilíaco-obturatória. Três doentes apresentaram comprometimento de ambas as cadeias. O número de linfonodos comprometidos variou de dois a sete com mediana de três. Mediana de tempo de seguimento foi

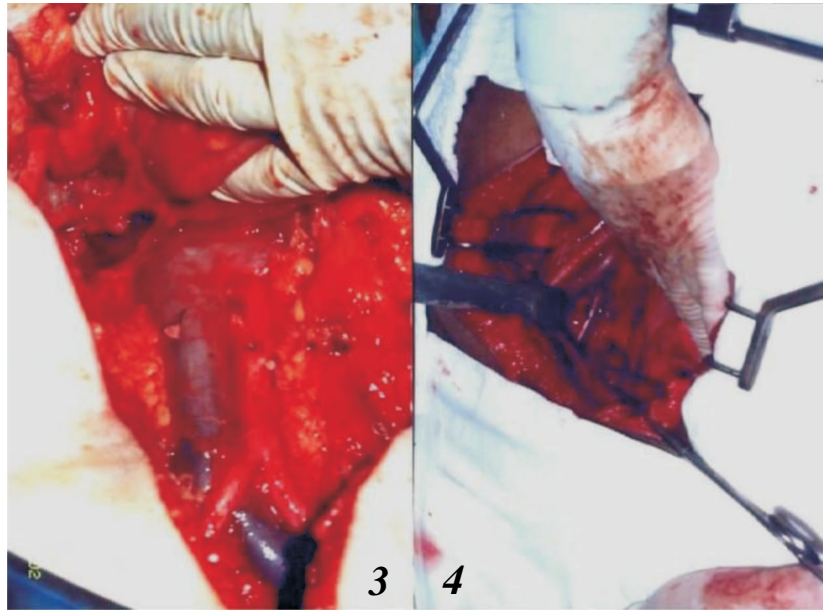

Figuras 3 e 4 -Linfadenectomias para-aórtica (à esquerda) e ilíaco-obturatória (à direita).

de 42 meses ( 9 - 59) enquanto a mediana de sobrevida foi de 40 meses. A sobrevida global em três anos foi de $77 \%(\mathrm{n}=14)$, enquanto a sobrevida livre de doença em três anos foi de $44 \%(n=8)$. Nenhum doente apresentou sobrevida de cinco anos. Entre o grupo que não realizou citorredução ótima $(\mathrm{n}=13)$, a sobrevida variou de 1 a 18 meses com mediana de 13 meses. Nesse grupo não houve sobreviventes por mais de três anos. Os resultados em longo prazo estão demonstrados na Tabela 3.

Doze experimentaram alguma recorrência (66\%). O intervalo livre de doença variou de 7 a 50 meses com mediana de 20 meses. Os sítios de recorrência foram: peritônio $(n=10)$, pelve $(n=6)$, pulmão $(n=1)$ e abdome superior $(n=1)$.

Em cinco, dois sítios de recorrência foram observados. Todas que apresentaram linfonodos comprometidos desenvolverão algum tipo de recorrência e finalmente faleceram pela neoplasia. Entre as doentes que apresentaram recorrência nove foram reoperadas com o objetivo de uma citorredução secundária. Entretanto, duas conseguiram uma citorredução ótima.

O tempo de sobrevida depois de diagnosticada a recorrência variou de 2 a 38 meses com mediana de 10 meses. A doente com maior sobrevida foi uma que seguiu uma citorredução secundária ótima (26 meses). Em relação aos sintomas pré-operatórios, o índice de controle depois da cirurgia foi de 88, $23 \%(n=16)$. Todas as doentes apresentavam sintomas pré-operatórios significantes levando a baixa qualidade de vida. Quando questionadas em relação a sua condição préoperatória e pós-operatória, dezesseis doentes respon- 
Tabela 1 - Características das Doentes.

$\mathrm{N}=19$

\begin{tabular}{|c|c|c|}
\hline Mediana de Idade & $54(24-77)$ & \\
\hline \multicolumn{3}{|l|}{ Estádio (FIGO, 1989) } \\
\hline III-C & 17 & \\
\hline IV & 2 & \\
\hline \multicolumn{3}{|l|}{ Grau } \\
\hline I & 9 & \\
\hline II & 7 & \\
\hline III & 3 & \\
\hline \multicolumn{3}{|l|}{ Tipo Histológico } \\
\hline Seroso & 15 & \\
\hline Mucinoso & 2 & \\
\hline Endometrióide & 2 & \\
\hline Celúlas Claras & 2 & \\
\hline \multicolumn{3}{|l|}{$A S A$} \\
\hline I & 12 & \\
\hline II-III & 5 & \\
\hline \multicolumn{3}{|l|}{ Sintomas } \\
\hline Massa Pélvica Palpável & 17 & \\
\hline Obstipação & 16 & \\
\hline Dispepsia & 14 & \\
\hline Queixas Urinárias & 5 & \\
\hline Dor Pélvica & 4 & \\
\hline \multicolumn{3}{|l|}{ Localização do maior implante da doença } \\
\hline Pelve & 15 & \\
\hline Abdome (Superior) & 2 & \\
\hline Linfonodos & 2 & \\
\hline Mediana do volume de ascite & $1580 \mathrm{ml}$ & $(300-5.000)$ \\
\hline Mediana de albumina sérica (pré-operatória) & $3,3 \mathrm{ng} / \mathrm{dl}$ & $(2,3-\quad 4,6)$ \\
\hline Mediana de CA 125 sérico ( pré-operatório) & $419 \mathrm{ng} / \mathrm{dl}$ & $(37-1.513)$ \\
\hline
\end{tabular}

deram que houve um bom controle dos sintomas e melhora da qualidade de vida. Todas retornaram a suas atividades rotineiras. Duas doentes concluíram que não houve qualquer melhora. Todas as doentes que realizaram anastomose colorretal $(n=15)$ apresentaram continência para sólidos depois da cirurgia. A mediana de tempo livre de sintomas relacionados à doença foi de 20 meses.

\section{DISCUSSÃO}

A porcentagem de pacientes com câncer de ovário em estádio avançado que podem ser submetidas à citorredução ótima varia muito na literatura. Essa grande diferença é multifatorial e depende de fatores como: experiência cirúrgica, local de tratamento (centro de referência terciário) ou mesmo o entusiasmo com a cirurgia de citorredução.

Citorredução ótima é um dos mais importantes fatores prognósticos tanto de sobrevida livre de doença quanto de sobrevida global nas pacientes com câncer epitelial de ovário em estádios avançados. ${ }^{(15)}$ Para que seja atingida uma citorredução ótima nos casos de doença pélvica extensa que comprometa estruturas adjacentes ao trato genital feminino torna-se necessário na maioria das pacientes uma ressecção do retosigmóide ou mesmo do trato urinário inferior (parcial ou total). A ressecção anterior baixa em monobloco foi 
Tabela 2 - Cirurgias e Resultados.

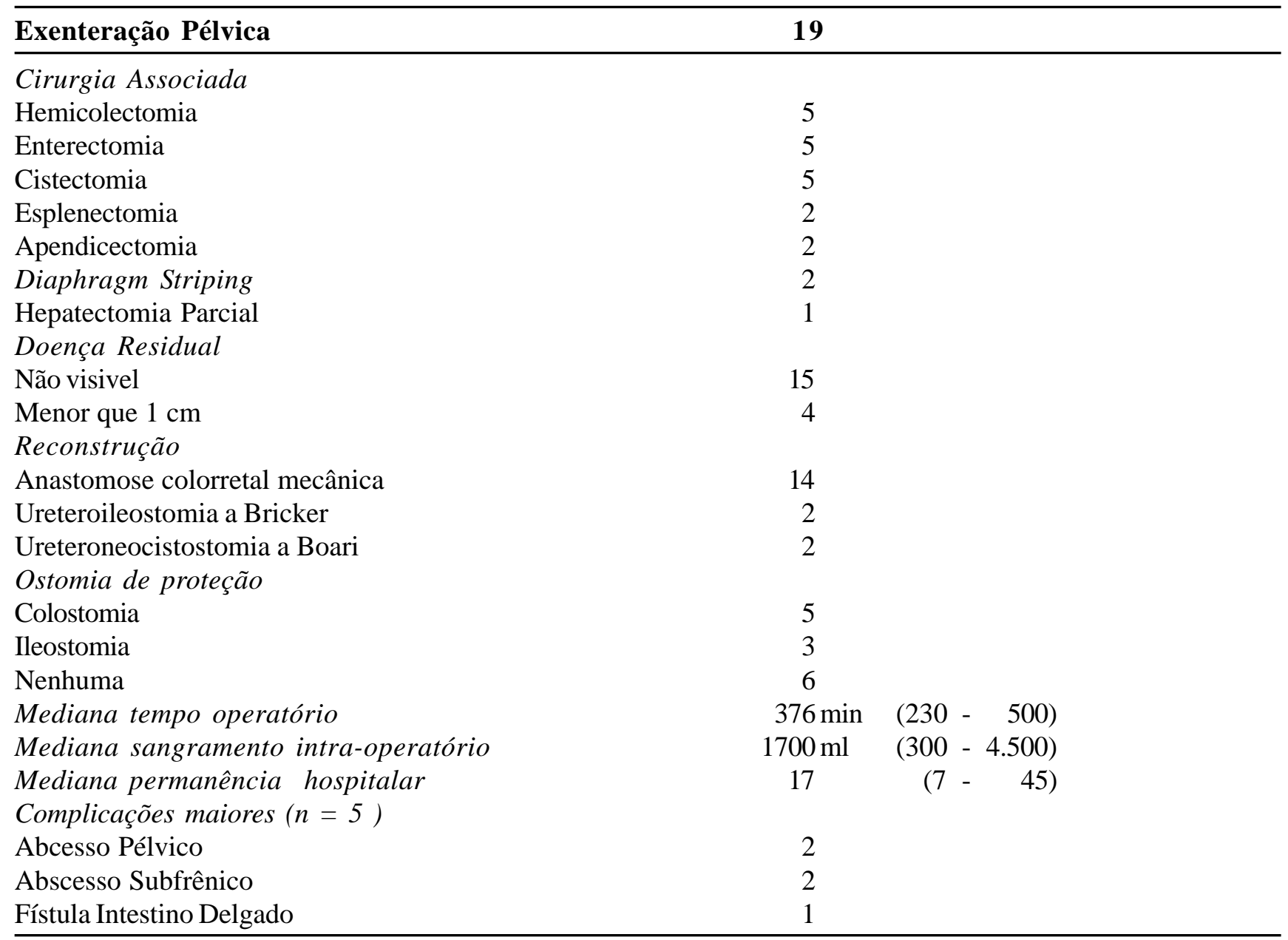

realizada em 16 a 58\% das pacientes com câncer de ovário nos estádios III-IV da FIGO. ${ }^{(15-19)}$

Ao utilizar a técnica descrita como exenteração pélvica posterior modificada, Eisenkop et al. ${ }^{(5)}$ conseguiram citorredução ótima (sem doença residual macroscópica) na pelve, em 18 de 47 (38,8\%) pacientes com câncer de ovário avançado. Após uma mediana de tempo de seguimento de 13,3 meses em $15(40,5 \%)$ das pacientes primariamente operadas não foi observada recorrência pélvica. Ao estender seu estudo inicial, Eisenkop et al. ${ }^{(20)}$ avaliaram 163 pacientes com câncer de ovário avançado e realizaram exenteração pélvica posterior modificada com anastomose retal baixa em 85 pacientes $(52,1 \%)$ com sucesso. Em 139 desses 163 pacientes $(85,35 \%)$ todo implante tumoral visível macroscopicamente fora removido. Benedetti-Panici et al. ${ }^{(21)}$ ao descreverem a
Tabela 3 - Resultados em Longo Prazo.

\section{N=19 (Citorredução ótima com exenteração)}

Sobrevida Global

3 anos

$77 \%$

5 anos

$0 \%$

Sobrevida livre de doença

3 anos

$44 \%$

5 anos

$0 \%$

N= 18 ( Sem citorredução ótima)

Sobrevida Global

3 anos

$0 \%$

abordagem retroperitoneal para o tratamento do câncer de ovário, realizada em 66 de 147 pacientes com neoplasia avançada (45\%), verificaram que a 
Os Benefícios da Ressecção Anterior Baixa em Monobloco para o Câncer de Ovário Avançado: Dez Anos de Experiência em um Único Centro Terciário Sergio Renato Pais Costa e Cols.
Vol. 28 ressecção colorretal foi necessária em 14 de 66 pacientes $(21 \%)$.

Com essa abordagem estes autores puderam atingir uma citorredução ótima (menos de $5 \mathrm{~mm}$ de maior implante tumoral residual) em 60 pacientes (91\%). Tais estudos demonstraram o importante papel das ressecções multiviscerais pélvicas (exenteração) na obtenção de uma citorredução ótima nas pacientes com câncer de ovário avançado com o objetivo de aumentar sua sobrevida. No presente estudo também pôde se observar que a exenteração pélvica proporcionou citorredução ótima em todas as doentes, sendo que na maioria não se observou doença macroscópica ao término da operação ( $78 \%$ cirurgia sem resíduo macroscópico). No entanto, em nosso ponto de vista, essa extensa operação somente permanece justificada quando todo o volume tumoral tanto da pelve quanto do abdome superior pode ser totalmente citorreduzido a menos do que $1 \mathrm{~cm}$ de doença residual. Mesmo assim, essa conduta pode levar a considerável morbidade e significante mortalidade. A morbidade da presente série foi similar à descrita por outros autores. ${ }^{5-8}$ Realizamos um maior número de reintervenções $(23,52 \%)$, quatro doentes foram reoperadas na mesma internação. A despeito da pequena amostra, três desses casos $(75 \%)$ foram do grupo submetido à citorredução primária. Esse fato foi mais comumente encontrado nas pacientes submetidas a múltiplas ressecções de órgãos além da exenteração, incluindo cinco hemicolectomias, três cistectomias parciais e uma hepatectomia parcial entre outros procedimentos maiores.

Resultados similares foram observados por Mohamed et al. ${ }^{11}$ sinalizando que a citorredução de intervalo após quimioterapia neo-adjuvante, deva ser realizada quando existe a possibilidade de um procedimento alargado com múltiplas ressecções e anastomoses para se atingir uma citorredução ótima. As complicações maiores dessa série como abscessos intra-cavitários devido às fístulas tanto de anastomose colorretal quanto de intestino delgado, também têm sido descritas por outros autores quando se trata de citorredução ótima com ressecções multiviscerais. ${ }^{6,7}$

Um escape da anastomose colorretal baixa pode acontecer mesmo com a confecção de uma ostomia protetora. Na presente série, a porcentagem de fístula na anastomose colorretal baixa foi de $6 \%$ similar ao encontrado por outros autores que variou de $0 \%$ a $14 \% .{ }^{21-27}$
Mesmo assim, essa fistula ocorreu em um caso extremo, onde a doente foi submetida a uma ressecção de múltiplos órgãos (ressecção do diafragma e esplenectomia) e apresentava o mais baixo valor de albumina sérica dessa série, bem como o maior sangramento intra-operatório $(4500 \mathrm{ml})$. Em séries reportadas previamente, é fato conhecido que tanto níveis baixos de albumina quanto grande número de transfusões estão associados a maior risco de fístula de anastomose. ${ }^{(6)}$ A despeito da drenagem percutânea de abscesso ser uma conduta de primeira escolha para o tratamento dos abscessos pélvicos ou abdominais acessíveis, nos três casos da presente casuística sua resolução foi por laparotomia. Contudo, esses casos foram dos mais antigos da série, quando a drenagem percutânea não era disponível e nem padronizada em nosso serviço. Paralelamente, alguns autores crêem que mesmo com uma drenagem percutânea adequada, uma reintervenção pode muitas vezes não ser evitada. ${ }^{(6,7)}$ Temos utilizado ostomia de proteção seletivamente para pacientes irradiados, quando a anastomose não fica segura ou é muito baixa (colo-anal). Assim sendo, nosso número de ostomias de proteção $(50 \%)$ foi próximo ao descrito por outros autores. ${ }^{(5,8)}$

Em nosso meio, poucas séries específicas de ressecção anterior baixa em monobloco (exenteração pélvica) têm sido descritas para o tratamento de neoplasias pélvicas avançadas.

Particularmente para o câncer de ovário avançado, não encontramos estudo similar no que tange seu tratamento com intenção curativa por meio de ressecções multiviscerais pélvicas (exenteração) como parte da estratégia de "Optimal Debulking".

Na presente série, o índice de citorredução ótima foi $56,66 \%$ similar aos resultados mais recentemente publicados por outros autores que têm variado de $50 \mathrm{a}$ $75 \% .^{5-28} \mathrm{~A}$ despeito da alta morbidade, a exenteração como parte da cirurgia de citorredução tem apresentado diminuição da mortalidade pós-operatória, mormente nas últimas séries que tem variado entre $0 \%$ e 6 $\% .^{5-30}$ No presente estudo a mortalidade foi similar inclusive com série descrita previamente pelo mesmo grupo para o tratamento do câncer de reto T4 que foi de $6 \% .{ }^{(10)}$ Mesmo considerando cirurgias abdominais extensas associadas à exenteração como parte da citorredução na maioria de nossos casos (90\%) similar ao descrito por Eisenhauer et al. ${ }^{(31)}$, a presente mortalidade pode ser considerada dentro do esperado na literatura. 
Os Benefícios da Ressecção Anterior Baixa em Monobloco para o Câncer de Ovário Avançado: Dez Anos de Experiência em um Único Centro Terciário Sergio Renato Pais Costa e Cols.
Vol. 28
Embora nenhum teste estatístico específico tenha sido feito no presente estudo, a mediana de sobrevida global das pacientes submetidas a ressecção anterior baixa em monobloco foi maior (42 meses X 13 meses) do que a daquelas que não a seguiram (quimioterapia exclusiva, citorredução subótima, tratamento paliativo). E paralelamente, a sobrevida global em três anos foi maior de $77 \%$ contra $0 \%$. Por outro lado, a sobrevida livre de doença no mesmo grupo foi de $44 \%$, ou seja, houve uma alta recorrência principalmente no peritônio ou na pelve. Sendo que não houve sobreviventes em cinco anos, mesmo para aquelas submetidas à ressecção anterior baixa em monobloco com citorredução ótima.

Nossos resultados são semelhantes aos observados por Buttarelli et al. ${ }^{(6)}$ que observaram uma mediana de sobrevida de 36,6 meses. Mais recentemente, Park et al. ${ }^{(30)}$ também observaram 30 meses de sobrevida mediana para doentes submetidas a esse tipo de cirurgia. Esses últimos observaram uma sobrevida em três anos de $82,03 \%$, para o grupo submetido a ressecção anterior baixa em monobloco com citorredução ótima contra $66,63 \%$ nas doentes não submetidas a essa técnica $(\mathrm{p}=0.04)$. Assim sendo, esses achados suportam a realização de uma cirurgia pélvica extensa com ressecção multivisceral como parte do tratamento do câncer de ovário avançado, principalmente quando uma citorredução ótima pode ser exequíivel.
Apesar de ser conduta de exceção, além de proporcionar um prolongamento da sobrevida pode também melhorar os sintomas importantes do câncer de ovário avançado. A obstipação, o tenesmo ou a dor pélvica podem ser temporariamente controlados com essa técnica podendo assim acarretar uma melhor qualidade de vida para esses doentes..$^{5-8}$ Embora não tenha sido elaborado um questionário sobre qualidade de vida no presente estudo, pode-se observar um melhor controle temporário dos sintomas na maioria das pacientes operadas. Similar a Butarelli et al. ${ }^{(6)}$, a preservação da continência fecal foi possível em todas doentes submetidas à anastomose colorretal baixa, melhorando assim a sua qualidade de vida. Todas as doentes retornaram a suas atividades diárias. Os sintomas préoperatórios foram bem controlados em $88 \%$ das doentes, durante uma mediana de tempo livre dos sintomas de 20 meses.

\section{CONCLUSÃO}

Em suma, a ressecção anterior baixa em monobloco como parte da cirurgia citorredutora para o tratamento do câncer epitelial do ovário avançado não deve ser um obstáculo se a citorredução ótima é factível. Apresenta alta morbidade, porém baixa mortalidade. Pode conferir um adequado controle da doença em longo prazo, à custa de uma alta recorrência tardia. Paralelamente, os sintomas pré-operatórios também podem ser temporariamente controlados.

ABSTRACT: Introduction - The cornerstone of ovarian cancer treatment has been the association of debulking surgery with platinum based chemotherapy. When this neoplasm invades adjacent pelvic organs, in order to attain an optimal debulking should be performed an en bloc multivisceral pelvic resection. Aim - The objectives of this study were to assess the safety, efficacy and impact on survival of low anterior en bloc resection for primary advanced epithelial ovarian cancer. Methods - Complete follow-up data were available on 19 patients who underwent this surgical procedure for advanced ovarian cancer between January 1996 and June 2006. These subjects presented a median age of 58 years (24-77). All patients underwent optimal cytoreduction (less than $1 \mathrm{~cm}$ of residual disease). Results - The postoperative mortality was $5 \%(\mathrm{n}=1)$ while overall major morbidity was 26,3 $\%(n=5)$. The overall three-year survival rate was $77 \%$ while median of survival time was $\mathbf{4 2}$ months. Conclusions - Low anterior en bloc resection, when it is necessary must not be obstacle to obtain an optimal surgery. In despite its high morbidity presents acceptable mortality. Long-term disease control may be attained with this approach.

Key words: Pelvic Exenteration, Ovarian Cancer, Morbidity, Mortality, Treatment Outcome.

\section{REFERÊNCIAS}

1. Karlan BY, Markman MA, Eifel PJ. Gynecologic Cancers: Ovarian cancer, Peritoneal carcinoma, and Fallopian Tube carcinoma. In: DeVita VT, Helman S, Rosenberg SA eds. Cancer:
Principles \& Practice of Oncology, 7 th Edition, Philadelphia: Lippincot \& Wilkins; 2005. pp. 1240-65.

2. Hensley Ml, Alektiar KM, Chi DS. Ovarian and fallopiantube cancer. In: Barakat RR, Bevers MW, Gershenson DM, Hoskins WJ eds. MD Anderson Cancer Center \& Memorial 
Os Benefícios da Ressecção Anterior Baixa em Monobloco para o Câncer de Ovário Avançado: Dez Anos de Experiência em um Único Centro Terciário

Sergio Renato Pais Costa e Cols.
Vol. 28
Sloan-Kettering Cancer Center Handbook of Gynecologic Oncology, 1 Th Edition, London: Martin Dunitz; 2000. pp. 243-263.

3. Hoskins WJ, Lawton FG. Primary Cytoreductive Surgery for Advanced-Stage Disease. In: Gershenson DM, McGuire WP editors. Ovarian Cancer Controversies in Management, 1 th Edition, New York: Chuchill Livingstone; 1998. pp. 65-84.

4. Slomovitz BM, Soliman PT, Wolf JK. Gynecologic Cancers. In: Feig BW, Berger DH, Fuhrman GM editors. The M.D. Anderson Surgical Oncology Handbook, 4 th Edition, Philadelphia: Lippincott Williams \& Wilkins; 2006. pp. 520563.

5. Eisenkop SM, Nalick RH, Teng NNH. Modified Posterior Exenteration for Ovarian Cancer. Obstetrics \& Gynecology 1991; 78 (5): 879-85.

6. Butarelli M, Houvenaghel G, Leliévre L, Jacquemier J, Guimarand J, Delpero JR. Pelvic posterior exenteration with immediated colo-rectal anastomosis: Is it justified and feasible in advanced stage ovarian carcinoma? Ann Chir 2006; 131: 431-6.

7. Mourton SM, Temple LK, Abu-Rustum NR, Gemignani ML, Sonoda Y, Bochner BH, Brakat RR, Chi DS. Morbity of retosigmoidectomy resection and primary anastomosis in patients undergoing primary cytoreductive surgery for advanced epithelial ovarian cancer. Gynecol Oncol 2005; 99: 608-14.

8. Spirtos NM, Eisenkop SM, Schlaerth JB, Ballon SC. Secondlook laparotomy after modified posterior exenteration: Patterns of persistence and recurrence in patients with stage III and stage IV ovarian cancer. Am J Obstet Gynecol 2000; 182 (6): 1321-27.

9. Tamussino KF, Lim PC, Webb MJ, Lee RA, Lesnick TG. Gastrointestinal Surgery in Patients with Ovarian Cancer. Gynecol Oncol 2001; 80: 79-84.

10. Costa SRP, Antunes RCP, Paula RP, Pedroso MA, Farah JFM, Lupinacci RA. A Exenteração Pélvica para o Tratamento do Câncer de Reto Estádio T4: Experiência em 15 casos operados. Arq Gastroenterol 2007; 43 (4): no prelo.

11. Vergote I, Wever I, Tjalma W, Van Gramberen M, Decloedt J, Vam Dam P. Interval Debulking Surgery: An Alternative for Primary Surgical Debulking? Semin Surg Oncol 2000; 19: 4953.

12. Hegazy MAF, Hegazi RAF, Elsshafei MA, Setit AE, Elshamy MR, Eltatoongy M, Halim AAF. Neoadjuvant chemotherapy versus primary surgery in advanced ovarian carcinoma. WJSO 2005; 3: 57.

13. Staging announcement: FIGO Cancer Committee. Gynecol Oncol 1986; 25: 383-5.

14. Gallo A, Frigerio L. Neoadjuvant chemotherapy and surgical considerations in ovarian cancer. Curr Opin Obstet Gynecol 2003; 1: 25-31.

15. Bristow RE, Tomacruz RS, Armostrong DK, Trimble EL, Montz FJ. Survival effect of maximal cytoreductive surgery for advanced ovarian carcinoma during the platinum era: a meta-analysis. J Clin Oncol 2002; 20: 1248-59.

16. Hacker NF, Bereck JS, Lagasse LD, Nieberg RK, Elashoff RM. Primary cytoreductive surgery for epithelial ovarian cancer. Obstet Gynecol 1983; 61: 413-20.

17. Guidozzi F, Ball JH. Extensive primary cytoreductive surgery for advanced epithelial ovarian cancer. Gynecol Oncol 1994; 53: 326-30

18. Munkarah AR, Hallum AV $3^{\text {rd }}$, Morris M, Burke TW, Levenbeck C, Atkinson EN et al. Prognostic significance of residual disease in patients with stage IV epithelial ovarian cancer. Gynecol Oncol 1997; 64: 31-7.

19. Lichtenegger W, Sehouli J, Buchmann E, Karajenev C, Weideman H. Operative results after primary and secondary debulking operations in advanced ovarian cancer. J Obstet Gynaecol Res 1998; 24 447-51.

20. Eisenkop SM, Friedmann RL, Wang H. Complete cytoreductive surgery is feasible and maximizes survival in patients with advanced epithelial ovarian cancer: a prospective study. Gynecol Oncol 1998; 69: 103-8.

21. Benedetti-Panici, Maneschi F, Scambia G, Cutillo G, Greggi S, Mancuso S. The pelvic retroperitoneal approach in the treatment of advanced ovarian carcinoma. Obstet Gynecol 1996; 87: 103-8.

22. Obermair A, Hagenauer S, Tamandl D. Safety and efficacy of low anterior em bloc resection as part of cytoreductive surgery for patients with ovarian cancer. Gynecol Oncol 2001; 83: 115-20.

23. De la Cuesta RS, Goodman A, Halverson SS, Fuller Jr AF. En bloc pelvic peritoneal resection of the intraperitoneal pelvic viscera in patients with advanced epithelial ovarian cancer. Cancer J Sci Am 1996; 2: 152.

24. Bristow RE, del Carmen MG, Kaufman HS, Montz FJ. Radical oophorectomy with primary stapled colorectal anastomosis for resection of locally advanced epithelial ovarian cancer. J Am Coll Surg 2003; 197: 565-774.

25. Sonnendecker EW, Beale PG. Rectosigmoid resection without colostomy during primary cytoreductive for ovarian carcinoma. Int Surg 1989; 74: 10-2.

26. Clayton RD, Obermair A, Hammond IG, Leung YC, McCartney AJ. The Western Australian experience of the use of en bloc resection of ovarian cancer with concomitant rectosigmoid colectomy. Gynecol Oncol 2002; 84: 53-7.

27. Soper JT, Couchman G, Berchuck A, Clarke-Pearson D. The role of partial sigmoid resection for debulking epithelial ovarian carcinoma. Gynecol Oncol 1991; 41: 239-44.

28. Scarabelli C, Gallo A, Francheschi S. Primary cytoreductive surgery with rectosigmoid colon resection for patients with advanced epithelial ovarian cancer. Cancer 2000; 88: 389-97.

29. Hertel H, Diebolder H, Herrmann J, Kohler C, Kune-Heid R, Possover $\mathrm{M}$ et al. Is the Decision for Colorectal Resection Justified by Histopathologic Findings: A Prospective Study 
Rev bras Coloproct Abril/Junho, 2008
Os Benefícios da Ressecção Anterior Baixa em Monobloco para o Câncer de Ovário Avançado: Dez Anos de Experiência em um Único Centro Terciário

Sergio Renato Pais Costa e Cols.
Vol. 28 of 100 patients with Advanced Ovarian Cancer. Gynecol Oncol 2001; 83 (3): 481-4.

30. Park JY, Seo SS, Kang S, Lee KB, Lim SY, Choi HS, Park SY. The benefits of low anterior en bloc resection as part of cytoreductive surgery for advanced primary and recurrent epithelial ovarian cancer patients outweigh morbidity concerns. Gynecol Oncol 2006; 103 (3): 977-84.

31. Eisenhauer EL, Abu-Rustum NR, Sonoda Y, Levine DA, Poynor EA, Aghajanian $\mathrm{C}$ et al. The addition of extensive upper abdominal surgery to achieve optimal cytoreduction improves survival in patients with stages III-C-IV epithelial ovarian cancer. Gynecol Oncol 2006103 (3): 1083-90.
Endereço para correspondência: SERGIO RENATO PAIS COSTA Instituto de Oncologia São Paulo Avenida Pacaembu, 1400, CEP: 01234-200 Tel.: 11- 3666-2299

E-mail:sergiorenatopais@ig.com.br 